\title{
Towards a circulating marker of hepato-visceral fat excess: S100A4 in adolescent girls with polycystic ovary syndrome - Evidence from randomized clinical trials
}

\author{
Rita Malpique $^{1,3}$ | David Sánchez-Infantes ${ }^{2}$ | Cristina Garcia-Beltran ${ }^{1,3}$ | Siri D. Taxerås ${ }^{2}$ | \\ Abel López-Bermejo ${ }^{4}$ | Francis de Zegher ${ }^{5}$ | Lourdes Ibáñez ${ }^{1,3}$ (i)
}

\author{
${ }^{1}$ Endocrinology, Pediatric Research Institute \\ Sant Joan de Déu, University of Barcelona, \\ Barcelona, Spain \\ ${ }^{2}$ Endocrinology and Nutrition, Germans Trias i \\ Pujol Research Institute, Barcelona, Spain \\ ${ }^{3}$ Network Biomedical Research Center of \\ Diabetes and Associated Metabolic Disorders \\ (CIBERDEM), Health Institute Carlos III, \\ Madrid, Spain \\ ${ }^{4}$ Pediatric Endocrinology, Hospital Dr. Josep \\ Trueta \& Girona Institute for Biomedical \\ Research, Girona, Spain \\ ${ }^{5}$ Department of Development \& \\ Regeneration, University of Leuven, Leuven, \\ Belgium \\ Correspondence \\ L. Ibáñez, Hospital Sant Joan de Déu, \\ University of Barcelona, Passeig de Sant Joan \\ de Déu, 2, 08950 Esplugues, Barcelona, Spain. \\ E-mail: libanez@sjdhospitalbarcelona.org \\ Funding information \\ Instituto de Salud Carlos III; Fondo Europeo de \\ Desarrollo Regional, Grant/Award Numbers: \\ $\mathrm{PI} 15 / 01078$ and PI17/01455
}

\begin{abstract}
Summary
S100A4 is a marker of subcutaneous adipose tissue dysfunction. Polycystic ovary syndrome (PCOS) is often driven by hepato-visceral adiposity. PCOS phenotypes are normalized more by reduction of central fat with spironolactone/pioglitazone/metformin (SPIOMET) than by oral contraceptive (OC) treatment. We studied whether circulating S100A4 concentrations are high in adolescents with PCOS and, if so, whether they normalize more with $O C$ or SPIOMET. Assessments included circulating S100A4, endocrine markers, body composition, abdominal fat partitioning in controls $(n=12)$ and girls with PCOS ( $n=51$; age 15.8 y; body mass index [BMI] $24.5 \mathrm{~kg} / \mathrm{m}^{2}$ ), and 1-year changes in girls with PCOS randomized for OC $(n=27)$ or SPIOMET $(n=24)$ treatment. Mean S100A4 concentrations were $71 \%$ higher $(P<0.001)$ in girls with PCOS than in controls and associated with hepato-visceral adiposity $(r=0.47$; $P=0.001)$; S100A4 concentrations decreased more $(P<0.01)$ with SPIOMET, those decreases associating to hepato-visceral fat loss $(r=0.50 ; P<0.0001)$. S100A4 may become a circulating marker of hepato-visceral fat excess in adolescents with PCOS.
\end{abstract}

\section{KEYWORDS}

liver fat, PCOS, S100A4, visceral fat

\section{I INTRODUCTION}

S100A4 is a member of the $\mathrm{S} 100$ calcium-binding protein family and was first identified and categorized as a metastasis-associated protein. ${ }^{1}$ Subsequently, S100A4 was found to be involved in controlling cell growth, differentiation, survival, and migration in different cell types in physiological and pathological conditions, including inflammation, immune response, neuroprotection, and angiogenesis. ${ }^{2-4}$

Abbreviations: BMI, body mass index; HMW, high-molecular weight; HOMA-IR, homeostasis model assessment-insulin resistance; LDL-cholesterol, low-density lipoprotein cholesterol; MRI, magnetic resonance imaging; OC, oral contraceptive; PCOS, polycystic ovary syndrome; SPIOMET, spironolactone/ pioglitazone/metformin; WAT, white adipose tissue David Sánchez-Infantes should be considered joint first author.

Francis de Zegher and Lourdes Ibáñez should be considered joint last authors.
Recently, S100A4 was identified as an adipokine whose secretion by subcutaneous white adipose tissue (WAT) is increased in case of WAT dysfunction, for example, when there is subcutaneous adipocyte hyperthrophy, WAT inflammation, and/or insulin resistance, independently of body mass index (BMI). ${ }^{5}$

Polycystic ovary syndrome (PCOS) is a frequent cause of hirsutism and menstrual irregularity in adolescent girls and young women, is commonly accompanied by insulin resistance with compensatory hyperinsulinemia and hepato-visceral fat excess, independently of $\mathrm{BMI}^{6}{ }^{6}$ and is followed by comorbidities such as subfertility and type 2 diabetes. ${ }^{7,8}$ In nonobese adolescent girls with PCOS, a treatment with low-dose spironolactone, pioglitazone, and metformin (SPIOMET) normalizes the PCOS phenotype more broadly than does treatment with an oral contraceptive (OC) ${ }^{9}$ 
We studied whether circulating S100A4 concentrations are elevated in adolescents with PCOS and, if so, whether they are normalized more with OC or SPIOMET treatment, and whether they relate to central fat excess.

\section{I SUBJECTS AND METHODS}

Subsequent to the recent report on S100A4 in subcutaneous WAT, ${ }^{5}$ we have added circulating S100A4 as a secondary end point to the registration of two controlled trials (with essentially identical design) primarily exploring ovulation rates after randomized open-label treatment with OC versus SPIOMET in nonobese adolescent girls with PCOS; the first of these trials (ISRCTN29234515) has been completed, ${ }^{9}$ and the second (ISRCTN11062950) is scheduled to be completed in late 2019. Circulating S100A4 was measured in spare serum samples available from 22 (of 36) girls in the first trial, and from 29 (of 29) girls in the second trial (Figure S1), for a total population of 51 girls (average age $15.8 \mathrm{y}$; BMI $24.5 \mathrm{~kg} / \mathrm{m}^{2}$ ). Both trials were initiated in the Adolescent Endocrinology Unit of Sant Joan de Déu University Hospital, Barcelona, Spain. The inclusion criteria were hirsutism (score $>8$ on modified Ferriman and Gallwey scale), oligomenorrhea (menstrual intervals $>45 \mathrm{~d}$ ), gynaecological age $>2.0$ years, and absence of sexual activity (no need for contraception). Exclusion criteria were 21-hydroxylase deficiency; glucose intolerance or diabetes mellitus; evidence of thyroid, liver, or kidney dysfunction; hyperprolactinemia; and prior use of medications affecting gonadal or adrenal function, or carbohydrate or lipid metabolism. ${ }^{9}$ Mediterranean diet and regular exercise were recommended to all participating girls; OC treatment for 1 year consisted of $20 \mu \mathrm{g}$ of ethinylestradiol plus $100 \mathrm{mg}$ of levonorgestrel for $21 / 28$ days and placebo for 7/28 days; SPIOMET treatment for 1 year consisted of a low-dose combination ${ }^{9}$ of spironolactone $50 \mathrm{mg} / \mathrm{d}$, pioglitazone $7.5 \mathrm{mg} / \mathrm{d}$, and metformin $850 \mathrm{mg} / \mathrm{d}$.

Twelve age-matched, healthy girls (age 15.8 y) recruited in nearby schools served as controls. All had regular menstrual cycles, and none was hirsute or taking medication.

Birth weight and BMI (and their Z-scores) were retrieved, and endocrine-metabolic variables were assessed as described. ${ }^{9-11}$ Homeostasis model assessment-insulin resistance (HOMA-IR) was calculated as [fasting insulin in $\mathrm{mU} / \mathrm{L}$ ] $\times$ [fasting glucose in $\mathrm{mg} / \mathrm{dL}$ ]/405 Serum S100A4 was measured using a Human Protein S100A4 ELISA kit (CSB-EL02032HU, Cusabio Biotech, Wuhan, China) with a lower detection limit of $0.2 \mathrm{ng} / \mathrm{mL}$ and with intraassay and interassay variation coefficients of $<0.8 \%$ and $<10 \%$, respectively. Body composition was assessed by dual $\mathrm{X}$-ray absorptiometry with a Lunar Prodigy and Lunar software (version 3.4/3.5, Lunar Corp, Madison, Wisconsin); abdominal fat (subcutaneous and visceral) and hepatic fat were assessed by magnetic resonance imaging (MRI) using a multiple-slice MRI 1.5 Tesla scan (Signa LX Echo Speed Plus Excite, General Electric, Milwaukee, Wisconsin). ${ }^{9}$ Central fat was arbitrarily defined here as the sum of visceral fat (in $\mathrm{cm}^{2}$ ) and hepatic fat (in \%).

All statistical analyses were performed using SPSS version 23.0 (IBM, Armonk, New York). Baseline differences in S100A4 concentrations between controls and girls with PCOS were tested with unpaired $t$ test, adjusting for age and BMI. Longitudinal changes in quantitative variables between groups were compared by repeated-measures general linear model. Differences in longitudinal changes between groups were tested by the interaction term among between- and withinsubject effects. Associations between quantitative variables were sought by partial correlation analyses after adjustment for potentially confounding factors. $P<0.05$ was considered statistically significant. Data are presented as mean \pm standard error of the mean (SEM).

The studies were conducted after approval by the Institutional Review Board of Sant Joan de Déu University Hospital, after written informed consent by the parents, and after assent by each of the participants.

\section{3 | RESULTS}

The overall results are summarized in Table 1 . SPIOMET treatment was accompanied by more broadly normalizing effects than OC, including on circulating insulin, high-molecular weight (HMW) adiponectin, ultra-sensitive C-reactive protein (usCRP), and central (hepato-visceral) fat, as previously published. ${ }^{9}$

Figure $1 \mathrm{~A}$ and $1 \mathrm{~B}$ depicts that circulating S100A4 concentrations were $71 \%$ (or $2.1 \mathrm{Z}$-score) higher in girls with PCOS than in control girls and associated with hepato-visceral adiposity $(\mathrm{N}=63 ; r=0.47$; $P=0.001)$.

Figure $1 \mathrm{C}$ and $1 \mathrm{D}$ shows also that the elevated S100A4 concentrations in girls with PCOS decreased more with SPIOMET than OC treatment and that those decreases associated most closely (Table S1) with the reduction of hepato-visceral fat $(r=0.50 ; P<0.0001)$. One-year changes in circulating S100A4 correlated also with those in fasting insulin, HOMA-IR, low-density lipoprotein cholesterol (LDL-cholesterol), and HMW adiponectin (Table S1).

In multiple regression analysis, S100A4 and BMI were major determinants of central fat, together explaining $47 \%$ of its variance (beta: $0.419, P<0.0001$; beta: $0.505, P<0.0001$; respectively), adjusting for age as potential confounding factor.

\section{4 | DISCUSSION}

Adolescent girls with PCOS-diagnosed by the latest international criteria allowing for a relatively homogeneous phenotype ${ }^{12}$-were found to have elevated concentrations of circulating S100A4, which are lowered by a central fat reducing intervention with SPIOMET for 1 year. Baseline serum S100A4 levels in the total study population associated positively with central fat; in girls with PCOS, on-treatment changes in S100A4 associated most closely with those of markers of insulin resistance and central fat.

Adolescent PCOS appears to be essentially driven by an exhaustion of the capacity to expand subcutaneous WAT in a metabolically safe way, resulting in a lipotoxic state and in lipid deposition in central/ectopic depots (liver and viscera). ${ }^{13}$ In a study of adipose tissue from women with obesity, high presence of S100A4 has been associated with subcutaneous WAT dysfunction, inflammation and insulin resistance, and adipocyte hypertrophy, independently of $\mathrm{BMI} .{ }^{5}$ In contrast, S100A4 could be exerting a protective role in mice experiencing 
TABLE 1 Auxological, endocrine-metabolic, body composition, and abdominal fat partitioning assessments in girls with PCOS ( $\mathrm{n}=51$ )

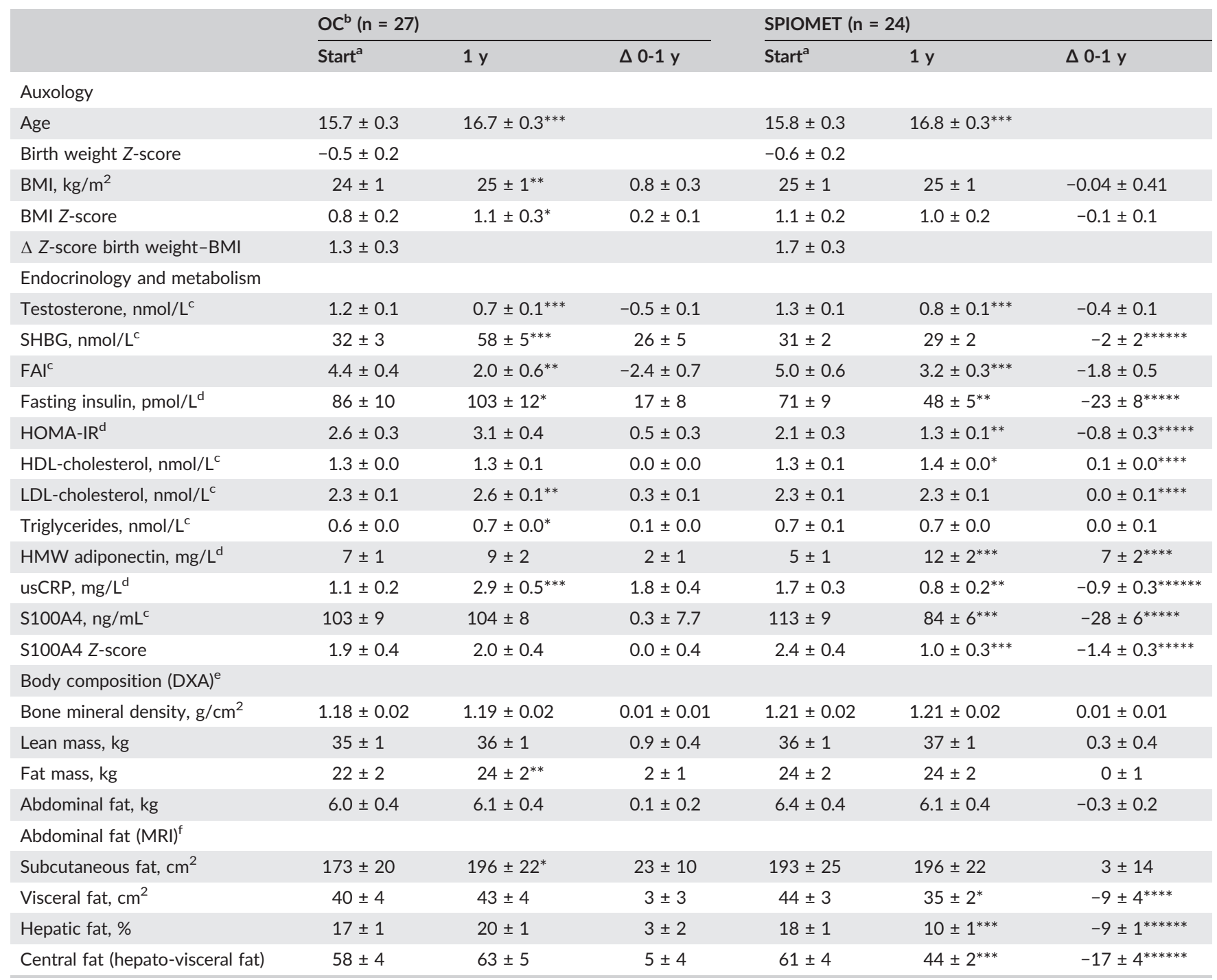

Abbreviations: BMI, body mass index; DXA, dual X-ray absorptiometry; FAI, free androgen index; HDL, high-density lipoprotein; HMW, high-molecular weight; HOMA-IR, homeostasis model assessment insulin resistance; LDL, low-density lipoprotein; MRI, magnetic resonance imaging; OC, oral contraceptive; PCOS, polycystic ovary syndrome; SHBG, sex hormone-binding globulin; SPIOMET, spironolactone, pioglitazone, and metformin; usCRP, ultra-sensitive C-reactive protein.

Values are mean \pm standard error of the mean (SEM).

$P$ values are adjusted for age and BMI.

${ }^{a}$ No significant differences between randomized subgroups at start.

${ }^{b}$ Ethinylestradiol $20 \mu \mathrm{g}$ plus levonorgestrel $100 \mathrm{mg}(21 / 28 \mathrm{~d})$.

'Indicative values in healthy adolescents $\left(\mathrm{n}=12\right.$; age $15.8 \mathrm{y}$; BMI $\left.23 \mathrm{~kg} / \mathrm{m}^{2}\right)$ : testosterone $0.9 \pm 0.1 ; \mathrm{SHBG} 61 \pm 4 \mathrm{nmol} / \mathrm{L}$; FAl $1.6 \pm 0.2 ; \mathrm{HDL}$-cholesterol $1.4 \pm 0.1 \mathrm{nmol} / \mathrm{L} ; \mathrm{LDL}$-cholesterol $2.4 \pm 0.2 \mathrm{nmol} / \mathrm{L}$; triglycerides $0.7 \pm 0.1 \mathrm{nmol} / \mathrm{L} ; \mathrm{S} 100 \mathrm{~A} 463 \pm 6 \mathrm{ng} / \mathrm{mL}$.

${ }^{\mathrm{d}}$ Indicative values in healthy adolescents $\left(\mathrm{n}=18\right.$; age $16.9 \mathrm{y} ; \mathrm{BMI} 21 \mathrm{~kg} / \mathrm{m}^{2}$ ): fasting insulin $21 \pm 2$ pmol/L; HOMA-IR $0.7 \pm 0.1 ; \mathrm{HMW}$ adiponectin $13 \pm 2 \mathrm{mg} / \mathrm{L}$; C-reactive protein $0.7 \pm 0.2 \mathrm{mg} / \mathrm{L}^{9}$

'Indicative DXA values in healthy adolescents, matched for age and height $(\mathrm{n}=41)$ : lean mass $35.1 \pm 1.0 \mathrm{~kg}$; fat mass $17.6 \pm 1.4 \mathrm{~kg}$. ${ }^{9}$

fIndicative MRI values in healthy adolescents $\left(\mathrm{n}=15\right.$; age $15.7 \mathrm{y}$; BMI $22 \mathrm{~kg} / \mathrm{m}^{2}$ ): subcutaneous fat $97 \pm 11 \mathrm{~cm}^{2}$; visceral fat $32 \pm 2 \mathrm{~cm}^{2}$; hepatic fat $12 \pm 1 \%{ }^{9}$

${ }^{*} P \leq 0.05$ for within-subgroup changes $(\Delta)$ from start.

${ }^{* *} P \leq 0.01$ for within-subgroup changes $(\Delta)$ from start.

${ }^{* * *} P \leq 0.001$ for within-subgroup changes $(\Delta)$ from start.

${ }^{* * * *} P \leq 0.05$ for between-subgroup changes $(\Delta)$ 0-1 year.

${ }^{* * * * *} P \leq 0.01$ for between-subgroup changes $(\Delta) 0-1$ year.

${ }^{* * * * * *} P \leq 0.001$ for between-subgroup changes $(\Delta) 0-1$ year. 
(A)

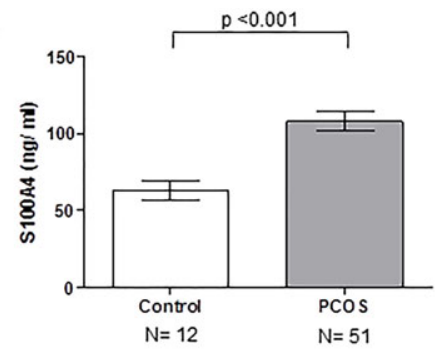

(B)

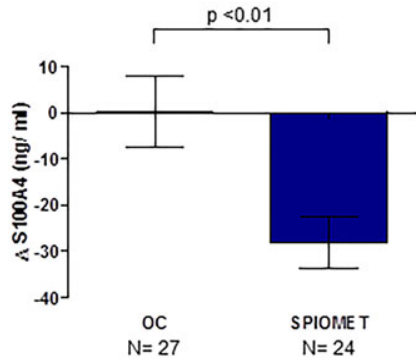

(C)

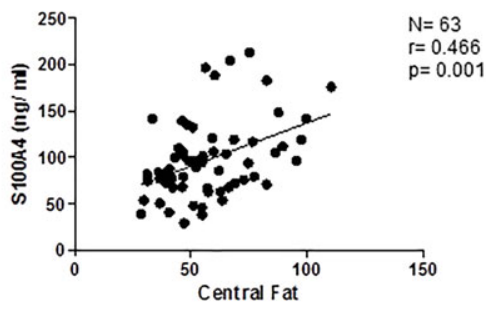

(D)

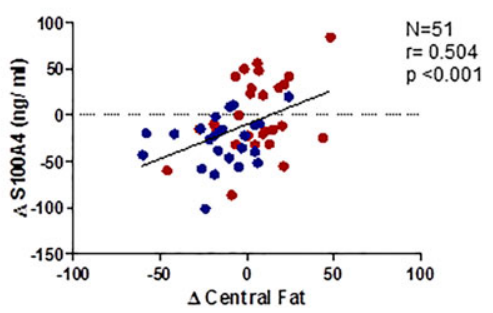

FIGURE 1 A, Baseline serum S100A4 concentrations in healthy controls $(n=12)$ and in girls with polycystic ovary syndrome (PCOS, $n=51)$. B, Correlation between baseline S100A4 levels and central fat in all subjects $(n=63)$. C, On-treatment $(0-1$ y) changes in circulating S100A4 concentrations after oral contraception (OC, $n=27$ ) or spironolactone, pioglitazone, and metformin (SPIOMET, $n=24$ ). D, Correlation between on treatment (0-1 y) changes in circulating S100A4 and changes in central fat in patients with PCOS $(n=51)$. Red dots correspond to girls receiving $O C(n=27)$; blue dots represent girls treated with SPIOMET $(n=24)$. Data are mean \pm standard error of the mean $(S E M)$. Central fat is the sum of visceral fat (by magnetic resonance imaging [MRI], in $\mathrm{cm}^{2}$ ) and hepatic fat (by MRI, in \%)

obesity and inflammation induced by a high-fat diet. ${ }^{14}$ Here, serum S100A4 was found to be linked to central fat; circulating S100A4 levels in girls with PCOS and central fat excess were increased as compared with healthy controls, and dropped to near-normal levels after insulin sensitization with low-dose SPIOMET, paralleling the fall in hepatovisceral fat, and the improvement of the markers of insulin resistance and adipose tissue inflammation and dysfunction. S100A4 could become a marker of central (hepato-visceral) fat excess, independently of whether the major origin of circulating S100A4 is subcutaneous or nonsubcutaneous adipose tissue. An inflammatory subset of macrophages in the liver may be an alternative or an additional source of circulating S100A4 in girls with hepatic fat excess ${ }^{15}$; a partially hepatic origin of circulating S100A4 in girls with PCOS would contribute to explain the fall of circulating S100A4 levels after SPIOMET-enhanced reduction of liver fatness, as well as the absence of such a fall after OC treatment, which fails to reduce liver fatness.

The present findings establish adolescent PCOS as condition combining central fat excess (even in the absence of obesity) with high circulating concentrations of S100A4, a protein that is long known to enhance the metastatic progression of a variety of cancers. These findings may be relevant in view of the evidence that young women with PCOS are at high risk (up to 18-fold) for developing endometrial carcinoma ${ }^{16}$ which happens to be the most BMI dependent among human cancers. ${ }^{17,18}$ If future studies indicate that chronic hepatovisceral adiposity and prolonged elevation of circulating S100A4 should no longer be viewed as innocent epiphenomena but as core PCOS features, then there may be a rationale to explore whether early SPIOMET-like interventions can reduce the incidence and/or improve the outcome of endometrial carcinoma in young women with PCOS ${ }^{19}$ and perhaps of other cancers related to hepatic and/or visceral adiposity. ${ }^{17,18}$ The new concept that S100A4 may serve as a key link between hepato-visceral fat excess and cancer merits further testing.

Study limitations include the relatively low number of participants, which may have prevented the detection of smaller differences between subgroups, and the absence of an untreated subgroup. Study strengths include the strict inclusion and exclusion criteria and the availability of a broad spectrum of advanced outcomes allowing to identify associations between circulating S100A4 and surrogates of adipose tissue dysfunction.

In conclusion, S100A4 may become a circulating marker of hepato-visceral fat excess, at least in adolescent girls with PCOS; the pathophysiological relevance of high concentrations of circulating S100A4 remains to be defined, in PCOS and also in other conditions.

\section{ACKNOWLEDGEMENTS}

This study was supported by a grant from the "Instituto de Salud Carlos III" and by the "Fondo Europeo de Desarrollo Regional" (FEDER), Madrid, Spain (PI15/01078 and PI17/01455).

R.M., C.G.-B., and L.I. are investigators of CIBERDEM (www. ciberdem.org). D.S.-I. is a Miguel Servet researcher (ISCIII, Ministry of Education and Science, Madrid, Spain), and A.L.-B. is an investigator of the 13 Fund for Scientific research (Ministry of Education and Science, Spain).

\section{CONFLICTS OF INTEREST}

No conflict of interest was declared. 


\section{AUTHORS' CONTRIBUTIONS}

R. Malpique researched and analysed data, contributed to data interpretation, and wrote the manuscript. D. Sánchez-Infantes contributed to study design, supervised the S100A4 assessments, and wrote the manuscript. C. García-Beltran researched and analysed data and contributed to data interpretation. A. López-Bermejo contributed to data analysis and interpretation, contributed to study design, and reviewed/edited the manuscript. D. Taxeras performed the S100A4 assessments. F. de Zegher contributed to study design and data interpretation and wrote/reviewed/edited the manuscript. L. Ibáñez contributed to study design, researched data, and wrote/reviewed/ edited the manuscript. Each author listed in the manuscript has seen and approved the submission of this version of the manuscript and takes full responsibility for the contents.

\section{ORCID}

Lourdes Ibáñez (D) https://orcid.org/0000-0003-4595-7191

\section{REFERENCES}

1. Ebralidze A, Tulchinsky E, Grigorian M, et al. Isolation and characterization of a gene specifically expressed in different metastatic cells and whose deduced gene product has a high degree of homology to a Ca2+-binding protein family. Genes Dev 1989; 3: 1086-1093.

2. Chen $M$, Sinha $M$, Luxon BA, Bresnick AR, O'Connor KL. Integrin alphabbeta4 controls the expression of genes associated with cell motility, invasion, and metastasis, including S100A4/metastasin. J Biol Chem 2009; 284: 1484-1494.

3. Gross SR, Sin CG, Barraclough R, Rudland PS. Joining S100 proteins and migration: for better or for worse, in sickness and in health. Cell Mol Life Sci 2014; 71: 1551-1579.

4. Fei F, Qu J, Li C, Wang X, Li Y, Zhang S. Role of metastasis-induced protein S100A4 in human non-tumor pathophysiologies. Cell Biosci 2017; 7: 64.

5. Arner P, Petrus P, Esteve D, et al. Screening of potential adipokines identifies S100A4 as a marker of pernicious adipose tissue and insulin resistance. Int J Obes (Lond) 2018 Jan 30 https://doi.org/10.1038/ s41366-018-0018-0.

6. Ibáñez L, Ong KK, López-Bermejo A, Dunger DB, de Zegher F. Hyperinsulinaemic androgen excess in adolescent girls. Nat Rev Endocrinol 2014; 100: 499-508.

7. Joham AE, Ranasinha S, Zoungas S, Moran L, Teede HJ. Gestational diabetes and type 2 diabetes in reproductive-aged women with polycystic ovary syndrome. J Clin Endocrinol Metab 2014; 99: E447-E452.

8. Hart R, Doherty DA. The potential implications of a PCOS diagnosis on a woman's long-term health using data linkage. J Clin Endocrinol Metab 2015; 100: 911-919.
9. Ibáñez L, del Río L, Díaz M, et al. Normalizing ovulation rate by preferential reduction of hepato-visceral fat in adolescent girls with polycystic ovary syndrome. J Adolesc Health 2017; 61: 446-453.

10. de Zegher F, Reinehr T, Malpique R, et al. Reduced prenatal weight gain and/or augmented postnatal weight gain predeces polycystic ovary syndrome in adolescent girls. Obesity 2017; 25: 1486-1489.

11. Malpique R, Bassols J, López-Bermejo A, et al. Liver volume and hepatic adiposity in childhood: relations to body growth and visceral fat. Int J Obes (Lond) 2018; 42: 65-71.

12. Ibáñez L, Oberfield SE, Witchel SF, et al. An international consortium update: pathophysiology, diagnosis, and treatment of polycystic ovarian syndrome in adolescence. Horm Res Paediatr 2017; 88: 371-395.

13. de Zegher F, Lopez-Bermejo A, Ibáñez L. Central obesity, faster maturation, and 'PCOS' in girls. Trends Endocrinol Metab 2018; 29: 815-818.

14. Hou S, Jiao Y, Yuan Q, et al. S100A4 protects mice from high-fat dietinduced obesity and inflammation. Lab Invest 2018; 98: 1025-1038.

15. Österreicher $\mathrm{CH}$, Penz-Österreicher M, Grivennikov SI, et al. Fibroblast-specific protein 1 identifies an inflammatory subpopulation of macrophages in the liver. Proc Natl Acad Sci U S A 2011; 108: 308-313.

16. Ding DC, Chen W, Wang JH, Lin SZ. Association between polycystic ovarian syndrome and endometrial, ovarian, and breast cancer: a population-based cohort study in Taiwan. Medicine 2018; 97: e12608.

17. Renehan AG, Zwahlen M, Egger M. Adiposity and cancer risk: new mechanistic insights from epidemiology. Nat Rev Cancer 2015; 15 : 484-498.

18. Giovannucci E. An integrative approach for deciphering the causal associations of physical activity and cancer risk: the role of adiposity. JNCI J Natl Cancer Inst 2018; 110: 935-941.

19. Mauland KK, Eng $\varnothing$, Ytre-Hauge $S$, et al. High visceral fat percentage is associated with poor outcome in endometrial cancer. Oncotarget 2017; 8: 105184-105195.

\section{SUPPORTING INFORMATION}

Additional supporting information may be found online in the Supporting Information section at the end of the article.

How to cite this article: Malpique R, Sánchez-Infantes D, Garcia-Beltran C, et al. Towards a circulating marker of hepato-visceral fat excess: S100A4 in adolescent girls with polycystic ovary syndrome - Evidence from randomized clinical trials. Pediatric Obesity. 2019;e12500. https://doi.org/ $\underline{10.1111 / \text { ijpo.12500 }}$ 